\title{
The Role of Penetrating Keratoplasty in the Management of Microbial Keratitis
}

\author{
COLIN M. KIRKNESS, LINDA A. FICKER, ARTHUR DMcG STEELE, \\ NOEL S. C. RICE \\ London
}

\begin{abstract}
Summary
Penetrating keratoplasty was performed as an emergency procedure in 52 eyes which had perforated from acute microbial keratitis and in a further 11 where perforation had not yet occurred. The results are compared with those of keratoplasty in 33 non-infected perforations and 20 eyes where there had been microbial keratitis which had responded to medical therapy leaving a scarred cornea. This latter group had both a better five year survival $(90 \%)$ compared to all the others $(51 \%), p<0.05$, and achieved significantly better visual acuities, $p<0.005$.
\end{abstract}

The aim of the management of microbial keratitis is to limit corneal destruction and so preserve or restore visual function. To this end, Jones ${ }^{1}$ discussed 'Decision making in microbial keratitis'. This wide-ranging review sought to establish guidelines for the coherent and logical diagnosis and management of corneal infections. Jones did not, however, discuss the role that surgery might play in this management strategy.

There can be no doubt that the best outcome for microbial keratitis is obtained when there is early presentation and when the disease process has not been modified by prior administration of antibiotics in an uncontrolled manner.

Occasionally, however, patients do present late or are referred after inadequate therapy. Sometimes, despite vigorous investigation, no organisms are satisfactorily identified. In these circumstances, inability to control the infection and the associated destructive inflammatory response may lead to invasion of the sclera or extensive tissue loss and per- foration. When small perforations occur, cyano-acrylate glue ('superglue') has been used as a management option ${ }^{2}$ but it is our experience that most cases will require keratoplasty to save the eye. Glue can be useful, however, at the time of keratoplasty by helping to maintain the anterior chamber while the graft bed is being prepared. ${ }^{2}$

This paper aims to discuss the role of penetrating keratoplasty in the decision-making process in the management of microbial keratitis and to define its relative success and complications. In order to put such surgery in context, three groups of patients will be presented, firstly those eyes in which there was active infection at the time of surgery; these have been subdivided into those eyes which had perforated and those which had not; secondly, as a control group, those eyes in which there was a sterile peforation whose aetiology was non-infective; and finally, as a further relevant control, those eyes which had been infected but which had responded to medical therapy and subsequently underwent

From the Pocklington Transplantation Unit \& Corneal Clinic, Moorfields Eye Hospital, London.

Correspondence to: Professor C. M. Kirkness, FRCS, FCOphth, Head of Department, Tennent Institute of Ophthalmology, University of Glasgow, 38 Church Street, Glasgow G11 6NT. 
penetrating keratoplasty for visual rehabilitation. This latter group forms an important part of the spectrum of microbial keratitis.

We further hope to establish factors associated with perforation in the presence of suppurative disease which influenced the outcome of keratoplasty.

It is important to bear in mind that these are a small and highly select group of the many more patients (approximately 200 patients per annum) whom we have treated at Moorfields for microbial keratitis.

\section{Patients and Methods}

Patients were identified from a database of the patients under the care of the Corneal Clinic, Moorfields Eye Hospital, but since 1985 , new cases of microbial keratitis undergoing keratoplasty have been followed prospectively. The total study period was from 1974-89. In those eyes with active uncontrolled infection or noninfected perforation, the indications for penetrating keratoplasty were primarily to preserve the globe. In the group in which there was active infection but in which perforation had not occurred, keratoplasty was undertaken either because perforation was threatened or because scleral extension of the abscess was imminent.

\section{Diagnostic groups}

A diagnosis of microbial keratitis was made on the basis of positive culture of an organism on at least one plate following initial corneal scrape, or biopsy at the time of keratoplasty. In addition, scrapes were also taken for microscopy eg Gram stain, Giemsa, acridine orange, or modified Zeil-Neelsen. Excised tissue was examined histologically for organisms. ${ }^{3}$ In some cases, the clinical pattern of suppurative keratitis was suggestive of a microbial infection but no organism was satisfactorily identified. These cases usually had received prior uncontrolled topical antibiotics. We refer to these as culture negative. ${ }^{3}$ Additional cases were due to necrotising keratitis in eyes with herpes simplex infections.

At the time of surgery, there was evidence of an acute infective or inflammatory process such as ulceration, infiltrates in the corneal stroma, keratic precipitates, anterior cham- ber flare or cells or hypopyon, or a positive isolate of an organism in 63 eyes, including 11 which had not perforated. We considered this to be active infection for descriptive purposes. There were 33 eyes which had perforated for reasons other than infection-the sterile perforation group.

There were 20 eyes in which microbial keratitis had been treated medically with eradication of the organism but in which there was considerable corneal scarring or vascularisation. For descriptive purposes we called these the inactive group.

\section{Surgery}

We did not attempt to use cyano-acrylate adhesive if a corneal or limbal perforation was greater than $1 \mathrm{~mm}$ in diameter. Conjunctival flaps were not used to seal perforations or to cover actively infected corneas.

The surgery involved in all these eyes followed the general principles of penetrating keratoplasty but several precautions and modifications of technique were used which may be germane to the outcome.

Great care was taken to excise all infected tissue. This was sent for further culture and for microscopic examination. We felt it was important to ensure that the wound edge was in healthy tissue (even if this meant having a graft considerably larger than the usual $7-8 \mathrm{~mm}$ ) to aid healing and prevent melting at the wound edge. At the same time, great care was taken to ensure that the donor button was large enough, bearing in mind that, in perforated eyes, a trephine may excise a block of tissue considerably larger than its nominal size. A whole eye stored in a moist chamber allowed much greater adaptability at this point. When the graft had to be very large, we aimed, wherever possible, to preserve part of the limbus to aid re-epithelialisation. ${ }^{4}$ This was achieved by siting the graft slightly eccentrically or by using a sector keratoplasty avoiding healthy limbal tissue. In contrast, in several patients with peripheral corneal disease, it was possible to avoid the visual axis altogether by performing a sector keratoplasty or eccentric tectonic graft.

The use of visco-elastic material was also helpful in delaminating the structures in the anterior chamber and so avoiding damage to 
the iris and underlying lens. Visco-elastic delamination was also helpful in opening up the angle and may have reduced the formation of anterior synechiae.

Clearance of inflammatory debris from the anterior chamber or vitreous was also attempted to speed visual rehabilitation and reduce post-operative inflammation. We did not remove the crystalline lens routinely unless there was an intumescent cataract. In some eyes which were very inflamed, prophylactic iridectomy was also performed to avoid pupil block even though synechiolysis and excision of anterior cyclitic membranes were attempted.

Post operatively, antibiotics appropriate for the infecting organism were used. These were changed depending upon laboratory culture and sensitivity results and upon the clinical response. If the eye was sterile, chloramphenicol drops were used. Topical steroids were given in high dosage beginning with a minimum of Gutt. Prednisolone $1 \%$ or equivalent every two hours (and often more) titrating the frequency and strength against the clinical response. Frequently patients were still taking Gutt Prednisolone 1\% four times a day at four months postoperatively and Prednisolone $0.3 \%$ once a day at one year. This regime was employed even when the infecting agent was known to be pseudomonas. This practice is justifiable on the basis that the infective tissue has been excised and the steroids are therefore unlikely to cause recrudescence.

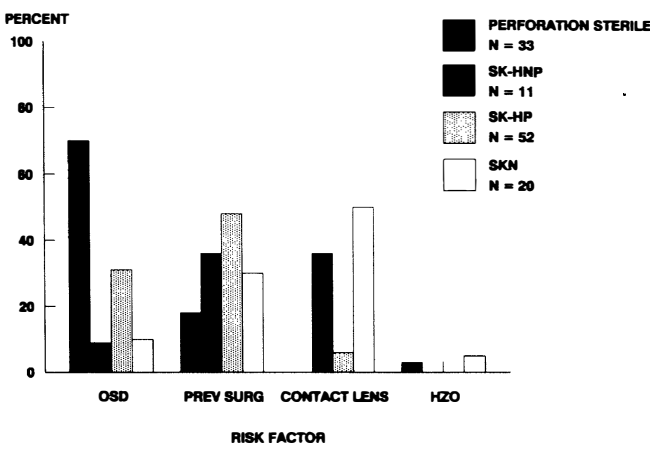

Fig. 1. The predisposing risk factors according for each of the study groups. Previous surgery consisted of glaucoma surgery in 2 cases, cataract surgery in 3 cases and 34 previous keratoplasties. SK-HNP-active infection, not perforated; $S K-H P$ active infection, perforated; SKN-inactive infection; OSD-ocular surface disease; HZO_herpes zoster ophthalmicus.
Mydriatics (gutt atropine) were used where there was inflammatory activity. Systemic steroids were not used regularly neither were prophylactic antiviral drugs in the case of an eye with a history of herpes simplex keratitis. Ancillary medications were employed where indicated.

Survival data were analysed according to the methods of Kaplan and Meier, and Gehan. ${ }^{56}$ Other statistical comparisons were made according to Student's t test and Chi squared testing.

\section{Results}

There were 116 keratoplasties in 95 eyes of 91 patients. All are under continuous review with the exception of three patients who died within three years of surgery. The follow-up is from six months to 13 years.

\section{Risk factors}

In 63 of the 83 infected cases $(76 \%)$ there was an obvious predisposing factor or more usually several factors eg surgery in eyes with severe ocular surface disease. Contact lens wear as also a common association in the infected cases. In contrast, ocular surface disease was more likely to be the aetiologic factor in the noninfected groups. (Fig. 1).

A variety of infecting organisms were identified with Gram positive bacteria being the most common. In only one patient was there multiple infection and in 12 cases, mostly those referred after indiscriminate broad spectrum treatment, we failed to identify an organism. Active herpes simplex virus infection was involved in 13 eyes. (Fig. 2).

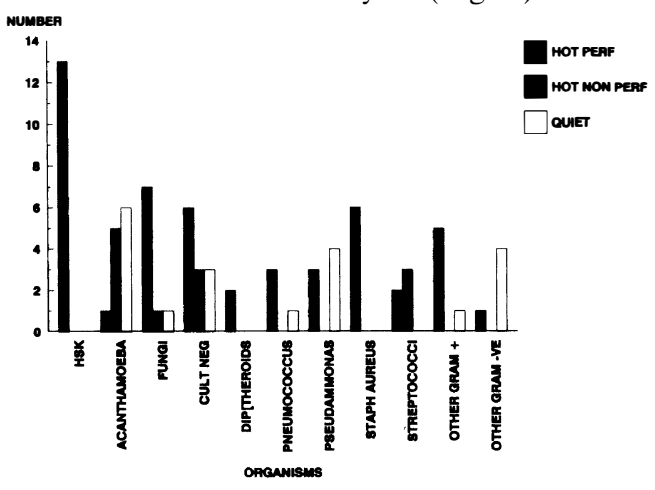

Fig. 2. The infecting organisms according to diagnostic groups. 'Cult neg' implies there was strong evidence of an infective agent eg organisms seen on Gram stain but there was no confirmatory culture. 


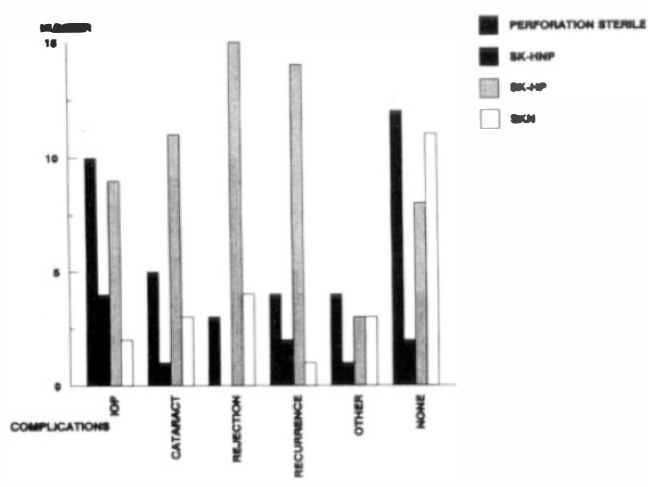

Fig. 3. The complications occurring in all eyes in the series. IOP implies that there was a sustained rise in pressure such that medical antiglaucoma treatment or glaucoma surgery was necessary. 'Recurrence' implies there was a further episode of microbial keratitis but in only one case was this the same as the original organism.

\section{Surgery}

The graft sizes ranged from $7 \mathrm{~mm}$ to $15 \mathrm{~mm}$, mean $8.51 \mathrm{~mm}$, median $8 \mathrm{~mm}$. Perforated eyes needed larger grafts-mean $8.55 \mathrm{~mm}$ compared to others $7.74 \mathrm{~mm}$ mean and the difference was statistically significant, $\mathrm{p}<0.05$. Only one case showed evidence of ongoing infection following keratoplasty, (vide infra) in all others, surgery was successful in eradicating the infection. Not surprisingly, a number of patients developed complications in the extended post-operative period. These are summarised in Fig. 3. Several patients had more than one complication and only 20 eyes had no serious complication.

Cataract occurred in 20 patients, 16 eyes have undergone successful cataract extraction, in the others surgery is deferred for medical reasons or because the patient does

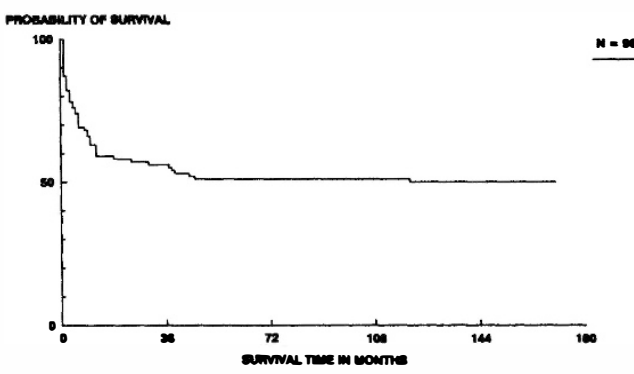

Fig. 4. The combined probability of survival of all the grafts for perforation in the series and those in actively infected eyes. not wish it. Glaucoma was a common problem, in 25 patients, all of whom had evidence of some degree of synechial angle closure, ranging from $60^{\circ}$ to total obliteration of the angle as judged by gonioscopy, post-operatively. Nine went on to have trabeculectomy and 10 had silicone drainage tubing inserted. ${ }^{7}$ Glaucoma had been present prior to keratoplasty in five of these patients.

Those in whom the perforation lasted more than 24 hours $(n=7)$ were much more likely to develop glaucoma than those in whom keratoplasty was performed within 24 hours, $\mathrm{p}<0.001$. Isolated rejection episodes were seen in 21 grafts.

The most serious complication was development of further infections-in 21 cases. The infecting organism was different from the original in all but one case. This cornea had perforated following keratomycosis due to Candida albicans and four months after keratoplasty developed another episode of keratomycosis again due to Candida albicans. This may represent failure of the surgery to erradicate the infective agent. In the other instances, the infection developed up to one year after the graft surgery and all had poor ocular surface environments. Three cases had endophthalmitis at the time of keratoplasty. These cases also showed resolution of the infection. No case developed endophthalmitis as an immediate post-operative complication.

Sixteen required further emergency keratoplasty.

\section{Survival and outcome}

Ultimately there were 43 failures, from rejection or from reinfection including one patient with Stevens-Johnson syndrome who had a primary conjunctival flap at the time of his

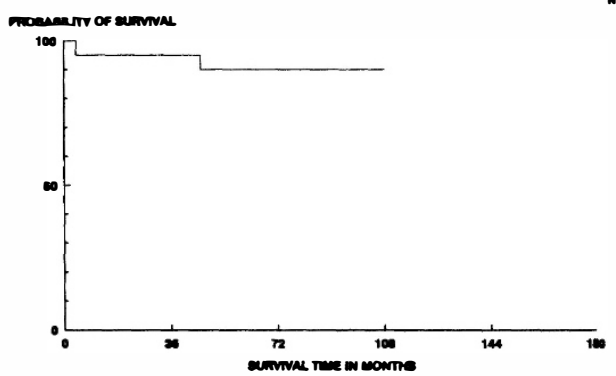

Fig. 5. The probability of survival of grafts performed on the inactive infected eyes. 


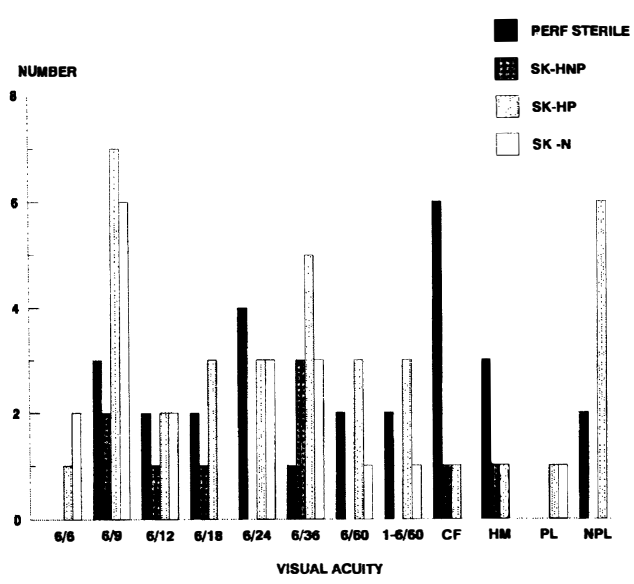

Fig. 6. Visual acuity following keratoplasty, according to diagnostic groups.

fourth graft. Five eyes were enucleated, eviscerated or became phthisical.

The overall probability of graft survival for those with active disease or infection at five years is $51 \%$. (Fig. 4). The perforated eyes with active infection, the sterile perforated eyes and the eyes with active infection which did not perforate all had similar graft survival and did not differ from one another statistically, $(\mathrm{p}>0.1)$. Only in the inactive group did the grafts have a significantly better survival, $(p<0.05)$ with a five year probability of graft survival of $90 \%$. (Fig. 5).

Forty eyes (34\%) obtained a final refracted acuity of $6 / 18$ or better and $29(31 \%)$ were worse than 6/60 including eight eyes which had no perception of light. The individual differences between each group is shown in Figure 6. The inactive group did significantly better than the others with $50 \%$ attaining $6 / 12$ or better, $\mathrm{p}<0.005$. Once again there was no difference between the other three groups.

\section{Discussion}

When one considers that the major indication for keratoplasty, in $91 \%$ of the eyes in this series, was to preserve the globe, the results are very encouraging and deserve to be viewed in that context.

\section{Graft survival}

The overall probability of graft survival at five years is $51 \%$. To put this in context, published series on all grafts for herpes simplex, show survival of about $60 \%$ at five years. ${ }^{8}$ Only the grafts in those eyes in which infection had been successfully treated and were inactive at the time of surgery had a better prognosis of about $90 \%$ at five years. There was no significant difference between the sterile perforated eyes and the infected perforated eyes. This suggests that perforation itself is a major risk factor and that an infecting organism seems to pose no hazard where it is treated with an appropriate antibiotic. Neither was there any significant difference between the perforated and unperforated, active infected sub-groups suggesting that the inflammatory sequelae of infection are important risk factors.

The improved survival of the successfully (medically) treated group may be explained, firstly, on the basis of the need for smaller grafts and secondly on the reduced inflammation in the eyes. In all of the emergency grafts, there was considerable intraocular inflammation. That such eyes grafted à chaud are at greater risk from rejection has been understood for many years. ${ }^{9}$ Many of the eyes did not, however, fail from rejection but failed due to problems related to ocular surface disorders, predisposing to further infections or recurrence of the original disease process such as peripheral conreal melting.

Only 20 eyes were free from serious complication. This figure emphasises the care which these eyes need in the post-operative period and the need for the patient to be able to be seen by an ophthalmologist immediately problems arise.

\section{Comparison with previous work}

Published work dealing with keratoplasty in microbial keratitis exists but direct comparisons are difficult. Kirkness ${ }^{10}$ in a small personal series showed an improved survival for those infected eyes which had not perforated, but this advantage is not sustained in this larger series from the same Institution. Hill ${ }^{11}$ reported the use of penetrating keratoplasty in the management of microbial keratitis in 23 patients in the active stage of the disease. He reported $70 \%$ with clear grafts at a mean follow-up of 20 months which is similar to our survival rate of about $65 \%$ at this time. $\mathrm{He}$ also reported similar complication rates of 
glaucoma, cataract and a reinfection rate of $25 \%$. Du et al. ${ }^{12}$ produced a very large series of emergency grafts for microbial keratitis and suggested good survival of the grafts for sizes less than $7 \mathrm{~mm}$ (only $14 \%$ failing) but when the graft size exceeded $8 \mathrm{~mm}, 79 \%$ failed. Unfortunately, they give little indication of the period of follow-up or the likely ongoing survival. They established the benefit of keratoplasty, at an early stage, in advanced suppurative disease. Complications encountered were similar to the present series.

Cooper and Constable ${ }^{13}$ likewise reported encouraging results in a small series of 13 patients, finding glaucoma and rejection the major problems. Their maximum follow-up was, however, only two years and only 4 were followed up to one year. Pouliquen et al. ${ }^{1+}$ in dealing with a diverse group of 33 keratoplasties for perforation found seven patients required a secondary keratoplasty, six of these being herpetic in origin. In $20 \%$ of patients $6 / 12$ acuity or better was achieved.

Beherens-Bauman ${ }^{15}$ reported the results of keratoplasty à chaud in 49 eyes. None of the eyes had to be enucleated. In $43 \%$, the transplant remained clear. The prognosis was best in cases of bacterial or fungal ulcers, provided that vascularisation did not involve more than one quadrant.

Recurrence of infections in vulnerable eyes has been reported previously. Fong et al. ${ }^{16}$ found that graft failure and HSK were both risk factors for recurrent infection and that more than half the infections occurred late. They were however, dealing with corneal transplants in general. Bates et al. ${ }^{17}$ recently demonstrated the risk factors, adverse ocular environment, loose sutures and failed grafts, for infections developing in corneal grafts. Several of their patients are included in this series which aims to report the total experience from the Corneal Clinic, Moorfields. Clearly, however, a corneal graft is itself risk factor for the development of microbial keratitis.

There is little published work specifically dealing with the problems of keratoplasty after sterile perforation but Volker and Nauman ${ }^{18}$ made an excellent case for the value of small (tectonic) graft avoiding the visual axis when there is a small peripheral perforation.
Our study suggests that the survival and complications of such grafts is similar to those done for infection.

\section{Recommendations}

On the basis of our results and the combined previous data we feel that there is a place for penetrating keratoplasty in the management of advanced microbial keratitis. Every effort should be made to identify the infecting agent and to modify the treatment regime in light of clinical response as suggested by Jones ${ }^{1}$ and Ficker et al. ${ }^{3}$ Tissue destruction may occur both as a result of uncontrolled infection and as a result of the induction of other lytic mechanisms. ${ }^{14}$ When perforation is imminent, our figures suggest that there is no advantage in waiting until an eye has perforated before proceeding to keratoplasty. Because the surgery may be technically easier, it may be better not to wait since, as Kirkness suggested, ${ }^{10}$ the angle may be better protected from peripheral synechial formation. Only further prospective study will reveal whether this belief is accurate.

We do not recommend delaying surgery once perforation has occurred since this leads to a greater risk of severe glaucoma. On the other hand, better results were obtained when infection was contained medically and keratoplasty delayed until the eye was quiet. We feel, however, the results of keratoplasty $\grave{a}$ chaud are sufficiently encouraging to suggest that penetrating keratoplasty should be undertaken in the first instance when perforation occurs or is threatened rather than fashioning a conjunctival flap as van $\mathrm{Rij}$ suggested. ${ }^{20}$

If there is a severe ocular surface disease which causes recurrence of infection so that a further keratoplasty is indicated, then there would seem to be grounds for considering a full conjunctival flap at the time of the second emergency keratoplasty since the survival of repeated emergency grafts was much reduced and in several cases the eye was ultimately lost. A conjunctival flap might have protected some of these vulnerable eyes from further problems and might have preserved the globes in four of the cases reported here. Acanthamoebic keratitis should be excluded from this argument since two keratoplasties 
may be needed to eradicate the organism completely. ${ }^{21}$

\section{Conclusion}

Eyes with severe suppurative keratitis or perforation can be preserved by emergency keratoplasty, with half maintaining a clear graft at five years. They may also attain useful visual acuities if the problem of rejection and reinfection can be avoided. These results can only be achieved by close co-operation between surgeon and patient so that complications are diagnosed promptly and managed optimally with attention to all aspects of the ocular and systemic pathology. It is worthwhile striving hard to save these eyes which might otherwise be lost.

Key words: penetrating keratoplasty; microbial keratitis; perforation; survival; complications.

\section{References}

${ }^{1}$ Jones DB: Decision making in microbial keratitis. Ophthalmology 1981, 88: 814-20.

${ }^{2}$ Maguen E, Nesburn B, Macy JI: Combined use of sodium hyaluronate and tissue adhesive in penetrating keratoplasty of corneal perforations. Ophthalmic Surg 1984, 15: 55-7.

${ }^{3}$ Ficker LA, Kirkness CM, Seal D, McCartney ACE: Microbial keratitis, the false negative: Eye (In press).

${ }^{4}$ Kirkness CM, Ficker LA, Steele ADMcG, Rice NSC: Large corneal grafts can be successful. Eye 1989, 3: 48-55.

${ }^{5}$ Kaplan E, Meier P: Non-parametric estimation from incomplete observations. J Am Statist Assoc 1958. 53: 457-81.

${ }^{6}$ Gehan EA: A generalised Wilcoxon test for comparing arbitrarily singly censored samples. Biometrika 1965, 52: 202-3.

${ }^{7}$ Kirkness CM, Ling Y, Rice NSC: The use of silicone drainage tubing to control post-keratoplasty glaucoma. Eye 1988, 2: 583-90.
${ }^{8}$ Ficker LA, Kirkness CM, Steele ADmcG, Rice NSC: The changing management of and improved prognosis in corneal grafting in herpes simplex keratitis. Ophthalmology 1989, 96: 1587-6.

${ }^{9}$ Szymankiewicz M, Weidle EG, Thiel HJ: Clinical aspects, course and results of keratoplasty a chaud. Fortschr Ophthalmol 1987, 84: 506-9.

${ }^{10}$ Kirkness CM, Steele ADMcG, Rice NSC: Penetrating keratoplasty in the management of suppurative keratitis. Dev Ophthalmol (Basel) 1989, 19: 172-5.

${ }^{11}$ Hill JC: Use of penetrating keratoplasty in acute bacterial keratitis. $\mathrm{Br} J$ Ophthalmol 1986, 70: 502-6.

${ }^{12}$ Du NZ, Chen JQ, Gong XM et al: Therapeutic keratoplasty in the management of purulent corneal ulceration-report of 100 cases. Jpn J Ophthalmol 1979, 23: 412-420.

${ }^{1.3}$ Cooper RL and Constable IJ: Draining pus from the cornea. Aust J Ophthalmol 1983, 11: 287-94.

${ }^{14}$ Pouliquen Y, Petroutsos G, Goichot L: Penetrating keratoplasty in corneal perforations. $\mathrm{J} \mathrm{Fr} \mathrm{Oph-}$ thalmol 1983, 6: 145-8.

${ }^{15}$ Behrens-Baumann W: Results of keratoplasty a chaud. Klin Monatsbl Augenheilkd 1984, 185: 25-76.

${ }^{16}$ Fong LP, Ormerod LD, Kenyon KR, Foster CS: Microbial keratitis complicating penetrating keratoplasty. Ophthalmology 1988, 95: 1269-75.

${ }^{17}$ Bates AK, Kirkness CM, Ficker LA et al: Microbial keratitis after penetrating keratoplasty. Eye 1990, 4: 74-8.

${ }^{1 *}$ Volker HE, Naumann GO: Eccentric tectonic minikeratoplaty in corneal, corneoscleral and scleral processes. Klin Monatsbl Augenheilkd 1984, 185: 158-66.

"Jones DB: Pathogenesis of bacterial and fungal keratitis. Trans Ophthalmol Soc UK 1978, 98: 367-71.

2) van Rij G, Rinkel van Driel B, Beekhuis WH, Renardel de Lavalette JG: Conjunctival transplantation. Doc Ophthalmol 1987, 67: 145-50.

${ }^{21}$ Ficker LA: Acanthamoeba; the quest for a better prognosis. Eye 1988, 2 (Suppl): 37-45. 